\title{
Clinical Evaluation of the Root Tear of the Posterior Horn of the Medial Meniscus in Total Knee Arthroplasty for Osteoarthritis
}

\author{
Eui-Sung Choi, MD and Sang-Jun Park, MD \\ Department of Orthopaedic Surgery, Chungbuk National University Hospital, Cheongju, Korea
}

\begin{abstract}
Purpose: To investigate the incidence of root tears of the posterior horn of the medial meniscus in total knee replacement arthroplasty for knee osteoarthritis and retrospectively analyze clinical results and factors associated with root tears.

Materials and Methods: There were 197 knees of 140 enrolled patients who had undergone total knee replacement arthroplasty between September 2010 and May 2014. The presence of a root tear of the posterior horn of the medial meniscus was confirmed in all patients. Statistical analysis was performed to investigate the correlation between root tears and the possible factors of meniscal tears including gender, age, severity of symptoms (visual analogue scale [VAS] score and medial joint line tenderness), grade of osteoarthritis (Kellgren-Lawrence grading scale), body mass index (BMI), varus deformity, and mechanical axis deviation.

Results: Meniscal tears were observed in 154 knees (78.17\%). The root tear had correlation with the severity of osteoarthritis $(\mathrm{p}<0.05)$, varus deformity $(\mathrm{p}<0.05)$, mechanical axis deviation $(\mathrm{p}<0.05)$, and BMI $(\mathrm{p}<0.05)$.

Conclusions: Factors considered to represent the severity of osteoarthritis were found to be associated with root tears of the medial meniscus posterior horn. Increased BMI seemed to be associated with the increased incidence of root tears of the medial meniscus posterior horn.
\end{abstract}

Keywords: Knee, Degenerative arthritis, Medial meniscus, Root tear, Arthroplasty

\section{Introduction}

The meniscus is a vital structure to normal knee function responsible for body weight distribution, shock absorption, proprioception, stabilization and lubrication of the knee joint, and pressure resistance ${ }^{1-5)}$. In particular, the weight-bearing function of the meniscus by circumferential hoop tension is directly associated with prevention of osteoarthritis of the knee ${ }^{6}$. In order to

Received November 3, 2014; Revised February 20, 2015;

Accepted April 2, 2015

Correspondence to: Eui-Sung Choi, MD

Department of Orthopaedic Surgery, Chungbuk National University

Hospital, 776 1(il)sunhwan-ro, Heungdeok-gu, Cheongju 361-711,

Korea

Tel: +82-43-269-6077, Fax: +82-43-274-8719

E-mail: oseschoi@chungbuk.ac.kr

This is an Open Access article distributed under the terms of the Creative Commons Attribution Non-Commercial License (http://creativecommons.org/licenses/by-nc/4.0/) which permits unrestricted non-commercial use, distribution, and reproduction in any medium, provided the original work is properly cited. maintain the circumferential hoop tension, the attachment of the anterior and posterior horns of the meniscus to the tibia should be secured and the meniscus collagen fiber orientation should be maintained $^{7)}$. However, a radial tear or root tear of the meniscus may lead to loss of circumferential hoop tension, which eventually results in osteoarthritis of the knee ${ }^{8)}$. A variety of recent research has focused on the loss of circumferential hoop tension as the main precursor of onset and progression of osteoarthritis ${ }^{9-11)}$. In this study, we retrospectively investigated the incidence of tears of the posterior root of the medial meniscus in patients who underwent total knee arthroplasty (TKA) for osteoarthritis and evaluated the relationship between clinical parameters and the root tears.

\section{Materials and Methods}

Between September 2010 and May 2014, TKA was performed for osteoarthritis on 210 knees (152 patients) at Chungbuk National University Hospital. Excluding those with a history of trauma (periarticular fracture of the knee or ligament injury 
requiring reconstruction or repair), infection, or rheumatoid arthritis, 197 knees (140 patients) were retrospectively reviewed for this study. The study population consisted of 17 males and 123 females with a mean age of 69.9 years (range, 53 to 89 years).

The presence of a tear of the posterior horn of the medial meniscus, which was defined as a complete radial tear or complex tear within $1 \mathrm{~cm}$ of the posterior horn insertion site of the medial meniscus, was confirmed in all patients (Fig. 1).

The relationships of the presence of posterior root tears of the medial meniscus with age and gender were analyzed. The Kellgren-Lawrence grading scale was used to classify the patients with respect to severity of osteoarthritis on radiography, and the incidence of root tears of the meniscus was compared between groups. The severity of varus deformity was measured on standing anteroposterior radiographs. Mechanical axis deviation was assessed with reference to a line drawn from the center of the femoral head to the center of the ankle on the long cassette view (Fig. 2). Body mass index (BMI) was assessed to classify the patients into four subgroups (underweight, normal weight, overweight, and obese groups) for comparison with regard to the prevalence of meniscal tears. Medial joint line tenderness was examined during a preoperative physical examination in our outpatient clinic and assessed using visual analogue scale (VAS).
In bilateral TKA patients, the incidence of unilateral/bilateral meniscal tears was assessed and the total number of tears was calculated on each side.

Statistical analyses were performed using the SPSS ver. 17.0 (SPSS Inc., Chicago, IL, USA). Chi-square tests and t-tests were

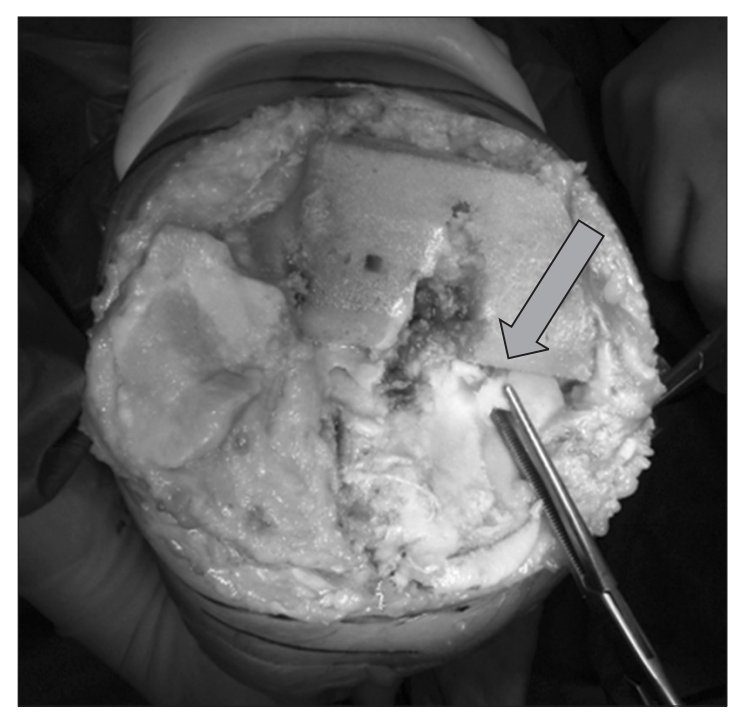

Fig. 1. Intraoperative photograph showing a root tear of the medial meniscus posterior horn.
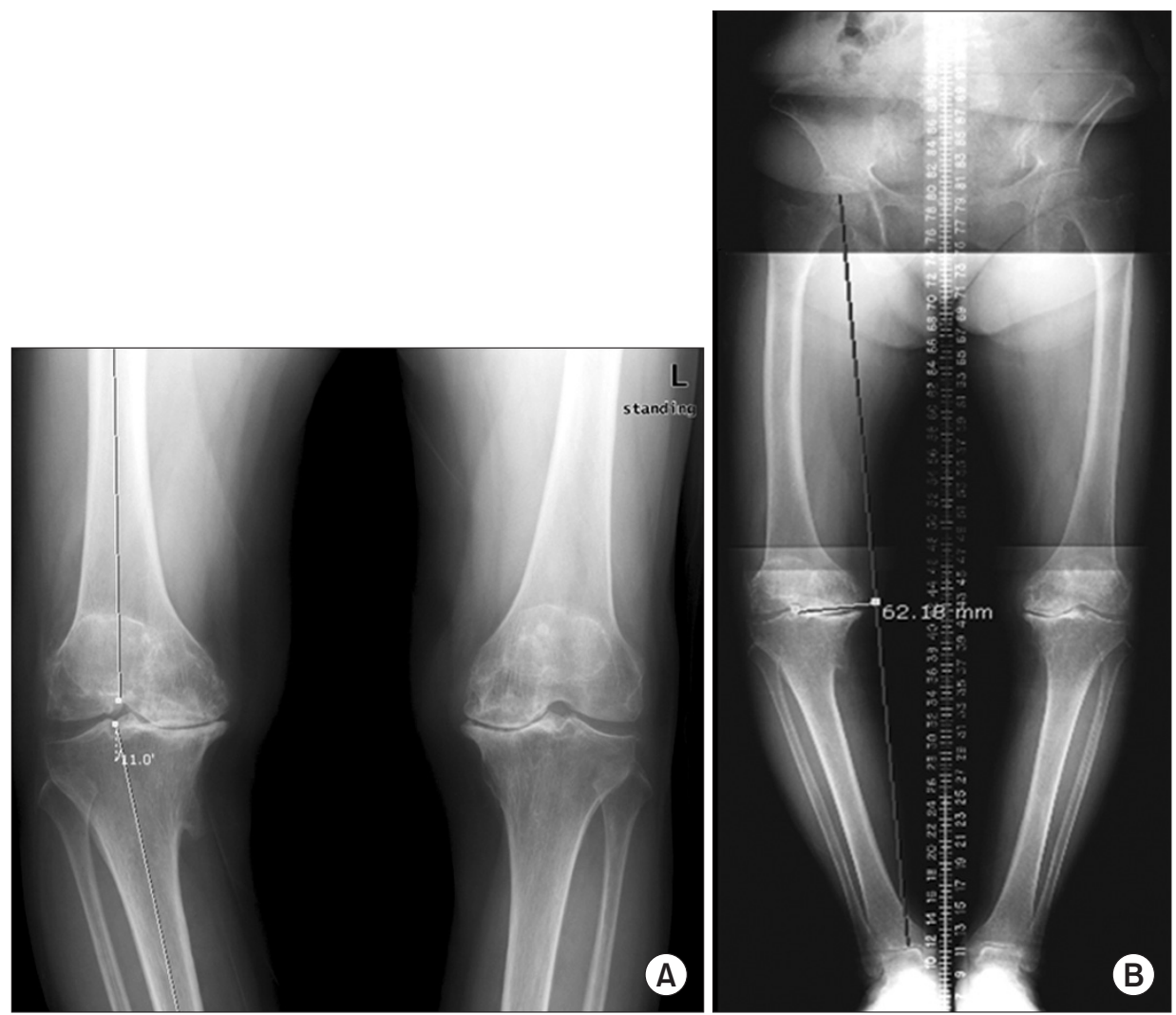

Fig. 2. (A) Anteroposterior standing radiograph of both knees showing the presence and degree of varus deformity. (B) Long cassette view for measurement of mechanical axis deviation. 
Table 1. The Incidence of Root Tears according to Age $(\mathrm{p}=0.946)$

\begin{tabular}{cccc}
\hline \multirow{2}{*}{ Age $(\mathrm{yr})$} & Total & \multicolumn{2}{c}{ Root tear } \\
\cline { 3 - 4 } & & $13(92.8)$ & 1 \\
\hline $50 \mathrm{~s}$ & 14 & $39(70.9)$ & 16 \\
$60 \mathrm{~s}$ & 55 & $92(80.0)$ & 23 \\
$70 \mathrm{~s}$ & 115 & $10(76.9)$ & 3 \\
$80 \mathrm{~s}$ & 13 & &
\end{tabular}

Values are presented as number (\%).

used to assess the relationship between meniscal tears and each parameter. Relationship with age and Kellgren-Lawrence grading scale was assessed using Fisher's exact test.

\section{Results}

Of the total 197 knees (140 patients) that were treated with TKA, 154 knees $(78.17 \%)$ had posterior root tears of the medial meniscus. The average age of the patients with a meniscal tear was 71.4 years (range, 56 to 86 years). The incidence of posterior root tears of the medial meniscus was highest in patients in their 50s: tears were observed in 13 of 14 knees in patients (92.8\%) in their 50s; in 39 of 55 knees in patients (70.9\%) their 60s; in 92 of 115 knees in patients (80\%) in their 70s; and in 10 of 13 knees in patients (76.9\%) in their 80s. However, the analysis results showed no significant relationship between meniscal tears and age $(\mathrm{p}=0.946)$ (Table $1)$.

Eleven of the 19 male knees (57.9\%) and 143 of the 178 female knees $(80.3 \%)$ had meniscal tears, indicating significant difference between the gender $(\mathrm{p}=0.038)$.

For the assessment of the relationship between BMI and posterior root tears of the medial meniscus, the patients were divided into 4 groups with regard to BMI: underweight $(<18.5)$, normal weight (range, 18.5 to $24.9 \mathrm{~kg} / \mathrm{m}^{2}$ ), overweight (range, 25.0 to $\left.29.9 \mathrm{~kg} / \mathrm{m}^{2}\right)$, and obese $(>30)$. There was no patient who fell into the underweight category. Meniscal tears were present in 33 of 48 knees $(68.8 \%)$ in the normal weight category, in 73 of 92 knees (79.4\%) in the overweight category, and in 48 of 57 knees (84.2\%) in the obese category, exhibiting a tendency of positive correlation between BMI and the incidence of meniscal tears. The mean BMI of patients with meniscal tears was 28.38, whereas the mean value of those without tears was 25.73. Thus, the t-test results showed a statistically significant relationship between the presence of root tears and BMI ( $\mathrm{p}=0.025)$.

According to the Kellgren-Lawrence grading scale, the severity of osteoarthritis based on plain radiography was grade III for 38 knees and grade IV for 159 knees. Posterior root tears of the me-
Table 2. The Incidence of Root Tears accoidng to Kellgren-Lawrence Grading Scale $(\mathrm{p}=0.017)$

\begin{tabular}{cccc}
\hline \multirow{2}{*}{$\begin{array}{c}\text { Kellgren-Lawrence } \\
\text { grading scale }\end{array}$} & Total & \multicolumn{2}{c}{ Root tear } \\
\cline { 3 - 4 } & & $(+)$ & $(-)$ \\
\hline III & 38 & $17(44.7)$ & 21 \\
IV & 159 & $137(86.2)$ & 22 \\
\hline
\end{tabular}

Values are presented as number (\%).

dial meniscus were more prevalent in grade IV knees: 17 in grade III knees (44.7\%) vs. 137 in grade IV knees (86.2\%). Thus, the severity of osteoarthritis confirmed with plain radiography was significantly related to the presence of meniscal tears $(\mathrm{p}=0.017)$ (Table 2).

Varus deformity was found to be significantly related to the presence of posterior root tears of the medial meniscus: the mean varus deformity was $13.56^{\circ}$ in knees with meniscal tears and $10.92^{\circ}$ in knees without meniscal tears $(\mathrm{p}=0.027)$. The mean medial deviation of the mechanical axis was $36.54 \mathrm{~mm}$ in the knees with meniscal tears and $30.17 \mathrm{~mm}$ in the knees without meniscal tears, showing a significant difference with regard to mechanical axis deviation ( $\mathrm{p}=0.043)$.

No significant relationship was found between posterior root tears of the medial meniscus and medial joint line tenderness $(\mathrm{p}=0.349)$ and VAS $(\mathrm{p}=0.466)$.

The surgery was bilateral in 116 knees (58 patients), of which meniscal tears were observed in 97 knees (83.6\%). The tear was located on the right side in 46 knees and on the left side in 51 knees, showing no notable difference $(\mathrm{p}=0.175)$. Meniscal tears were present in both knees in 42 patients (72.4\%) and on one knee in 13 patients (22.4\%; 4 left knees and 9 right knees). Three patients (5.2\%) had no meniscal tear on either side.

\section{Discussion}

The meniscus plays an important role in body weight distribution, shock absorption, proprioception, and stabilization and lubrication of the knee joint ${ }^{1-5)}$, all of which could become impossible if the meniscus is torn or displaced. A complete radial tear or complex tear at the posterior horn insertion site of the meniscus causes displacement of the meniscus, which may eventually lead to development of osteoarthritis ${ }^{12,13)}$. In other words, mechanical alterations in the knee joint due to increased load on a particular compartment in the knee result in chondral and subchondral damage.

Osteoarthritis of the knee was first described in 1968 by Ahlback et al. ${ }^{14)}$ who had conducted research on senior female 
patients. So far, the causes of knee osteoarthritis have been investigated in a variety of studies, and meniscal pathology has been particularly associated with the degenerative condition of the knee ${ }^{15-18)}$. Ahlback et al. ${ }^{14)}$ noted meniscal tears in 6 out of 14 osteoarthritis patients (42.9\%). Norman and Baker ${ }^{19)}$ observed meniscal tears in $50 \%-78 \%$ of their patients.

Since Pagnani et al. ${ }^{20)}$ documented arthroscopic evidence of accelerated degeneration of the articular cartilage in a 20 -year-old football player with a posterior root tear of the medial meniscus in 1991, attention has been brought to meniscal tears as the main precursor of the development of osteoarthitis. Robertson et al. ${ }^{21)}$ noted tears of the posterior root of the medial meniscus in $80 \%$ of their patients with osteoarthritis. Bin et al. ${ }^{9)}$ reported radial tears of the posterior horn accounted for $27.8 \%$ of the medial meniscal tears. Bessette ${ }^{2)}$ emphasized the importance of maintaining hoop tension to preserve the normal meniscus function, which decreases more drastically in cases of radial tears of the anterior or posterior horn than the mid body of the meniscus. Ozkoc et $\mathrm{al}^{22)}$ reported that posterior horn root tears are more frequent in the medial than lateral meniscus due to the greater mobility of the posterior horn attachment to the tibia in the lateral meniscus. Allaire et al. ${ }^{10)}$ described that the influence of posterior root tears of the medial meniscus on knee biomechanics was equivalent to that of meniscectomy in a cadaver model. They attributed loss of hoop tension of the meniscus resulting in the inability to distribute body weight and subluxation of the meniscus to greater contact pressure in the knee joint and early development of articular cartilage degeneration.

In the current study, based on a review of the abovementioned studies presenting posterior root tears of the medial meniscus as the main causative factor of osteoarthritis, we attempted to investigate the relationship between the meniscal tears and clinical factors.

Of the total 197 knees (140 patients), posterior root tears of the medial meniscus were observed in 154 knees (78.17\%). The incidence of meniscal tears was related to gender $(\mathrm{p}=0.376)$, but not to age $(p=0.946)$. There was a positive correlation between BMI and the presence of meniscal tears, and the mean BMI was significantly different between patients with tears and those without tears $(\mathrm{p}=0.025)$. However, considering that most of the patients were advanced age, the tears could have resulted from degenerative changes in the joint because BMI is correlated with the load on the medial compartment of the knee in advanced stage osteoarthritis. Factors potentially related to the severity of osteoarthritis, such as Kellgren-Lawrence grading scale, varus deformity, and medial deviation of the mechanical axis, showed significant relationship with posterior root tears of the medial meniscus. However, medial joint line tenderness and VAS were not found to be associated with meniscal tears. Our study results showed that the severity of osteoarthritis was correlated with the incidence of posterior root tears of the medial meniscus. In our opinion, the influence of meniscal tears on the progression of osteoarthritis could be confirmed in further arthroscopy or magnetic resonance imaging follow-up.

There were some limitations of this study. First, although the presence of a root tear was determined according to a standardized definition, it was difficult to avoid the influence of subjective judgement, and the causal relation between osteoarthritis and meniscal tears should be further verified. Second, the study was based only on the influence of medial meniscal tears on the progression of osteoarthritis; possible impact of accompanying meniscal tears was not considered as a variable in the analysis. In addition, the VAS score and medial joint line tenderness included in the analysis could have been affected by subjective judgement of the patient and clinician, respectively.

\section{Conclusions}

The presence of posterior root tears of the medial meniscus was significantly related to factors that have been associated with the severity of osteoarthritis, such as Kellgren-Lawrence grading scale, varus deformity, and mechanical axis deviation. Although no statistical significance was noted, there was a tendency of positive correlation between BMI and meniscal tears.

\section{Conflict of Interest}

No potential conflict of interest relevant to this article was reported.

\section{References}

1. Greis PE, Bardana DD, Holmstrom MC, Burks RT. Meniscal injury: I. Basic science and evaluation. J Am Acad Orthop Surg. 2002;10:168-76.

2. Bessette GC. The meniscus. Orthopedics. 1992;15:35-42.

3. Fukuda Y, Takai S, Yoshino N, Murase K, Tsutsumi S, Ikeuchi K, Hirasawa Y. Impact load transmission of the knee joint-influence of leg alignment and the role of meniscus and articular cartilage. Clin Biomech (Bristol, Avon). 2000;15: 516-21.

4. Insall JN, Scott WN. Surgery of the knee. 3rd ed. New York: 
Churchill Livingstone; 2001. p118-35.

5. Smillie IS. Injuries of the knee joint. 5th ed. New York: Churchill Livingstone; 1978. p112-88.

6. Grood ES. Meniscus function. Advs Orthop Surg. 1984;193-7.

7. Krause WR, Pope MH, Johnson RJ, Wilder DG. Mechanical changes in the knee after meniscectomy. J Bone Joint Surg Am. 1976;58:599-604.

8. Nha KW, Jo JH, Lee DB. Clinical results of the radial tear of posterior root of medial meniscus. J Korean Arthrosc Soc. 2007;11:128-33.

9. Bin SI, Kim JM, Shin SJ. Radial tears of the posterior horn of the medial meniscus. Arthroscopy. 2004;20:373-8.

10. Allaire R, Muriuki M, Gilbertson L, Harner CD. Biomechanical consequences of a tear of the posterior root of the medial meniscus: similar to total meniscectomy. J Bone Joint Surg Am. 2008;90:1922-31.

11. Lee JH, Lim YJ, Kim KB, Kim KH, Song JH. Arthroscopic pullout suture repair of posterior root tear of the medial meniscus: radiographic and clinical results with a 2 -year followup. Arthroscopy. 2009;25:951-8.

12. Lerer DB, Umans HR, Hu MX, Jones MH. The role of meniscal root pathology and radial meniscal tear in medial meniscal extrusion. Skeletal Radiol. 2004;33:569-74.

13. Fukubayashi T, Kurosawa H. The contact area and pressure distribution pattern of the knee. A study of normal and osteoarthrotic knee joints. Acta Orthop Scand. 1980;51:871-9.
14. Ahlback S, Bauer GC, Bohne WH. Spontaneous osteonecrosis of the knee. Arthritis Rheum. 1968;11:705-33.

15. Lotke PA, Ecker ML, Alavi A. Painful knees in older patients: radionuclide diagnosis of possible osteonecrosis with spontaneous resolution. J Bone Joint Surg Am. 1977;59:617-21.

16. Chen MI, Branch TP, Hutton WC. Is it important to secure the horns during lateral meniscal transplantation? A cadaveric study. Arthroscopy. 1996;12:174-81.

17. Shino K, Hamada M, Mitsuoka T, Kinoshita H, Toritsuka Y. Arthroscopic repair for a flap tear of the posterior horn of the lateral meniscus adjacent to its tibial insertion. Arthroscopy. 1995;11:495-8.

18. Shrive NG, O'Connor JJ, Goodfellow JW. Load-bearing in the knee joint. Clin Orthop Relat Res. 1978;(131):279-87.

19. Norman A, Baker ND. Spontaneous osteonecrosis of the knee and medial meniscal tears. Radiology. 1978;129:653-6.

20. Pagnani MJ, Cooper DE, Warren RF. Extrusion of the medial meniscus. Arthroscopy. 1991;7:297-300.

21. Robertson DD, Armfield DR, Towers JD, Irrgang JJ, Maloney WJ, Harner CD. Meniscal root injury and spontaneous osteonecrosis of the knee: an observation. J Bone Joint Surg Br. 2009;91:190-5.

22. Ozkoc G, Circi E, Gonc U, Irgit K, Pourbagher A, Tandogan $\mathrm{RN}$. Radial tears in the root of the posterior horn of the medial meniscus. Knee Surg Sports Traumatol Arthrosc. 2008; 16:849-54. 